ARTICLE

DOI: $10.1038 / s 41467-017-00666-6$

OPEN

\title{
Endoderm Jagged induces liver and pancreas duct lineage in zebrafish
}

Danhua Zhang ${ }^{1,2}$, Keith P. Gates ${ }^{1}$, Lindsey Barske ${ }^{3}{ }^{3}$, Guangliang Wang ${ }^{4}$, Joseph J. Lancman ${ }^{1}$, Xin-Xin I. Zeng ${ }^{1}$, Megan Groff ${ }^{3}$, Kasper Wang ${ }^{3}$, Michael J. Parsons ${ }^{4}$, J. Gage Crump ${ }^{3}$ \& P. Duc Si Dong1,2

Liver duct paucity is characteristic of children born with Alagille Syndrome (ALGS), a disease associated with JAGGED1 mutations. Here, we report that zebrafish embryos with compound homozygous mutations in two Notch ligand genes, jagged $1 b$ (jag $1 b$ ) and jagged $2 b$ (jag $2 b$ ) exhibit a complete loss of canonical Notch activity and duct cells within the liver and exocrine pancreas, whereas hepatocyte and acinar pancreas development is not affected. Further, animal chimera studies demonstrate that wild-type endoderm cells within the liver and pancreas can rescue Notch activity and duct lineage specification in adjacent cells lacking $j a g 1 b$ and jag $2 b$ expression. We conclude that these two Notch ligands are directly and solely responsible for all duct lineage specification in these organs in zebrafish. Our study uncovers genes required for lineage specification of the intrahepatopancreatic duct cells, challenges the role of duct cells as progenitors, and suggests a genetic mechanism for ALGS ductal paucity.

\footnotetext{
${ }^{1}$ Human Genetics Program, Sanford Burnham Prebys Medical Discovery Institute, 10901 North Torrey Pines Road, La Jolla, CA 92037, USA. ${ }^{2}$ Graduate School of Biomedical, Science, 10901 North Torrey Pines Road, La Jolla, CA 92037, USA. ${ }^{3}$ Department of Stem Cell Biology and Regenerative Medicine, Keck School of Medicine of University of Southern California, Los Angeles, CA 90033, USA. ${ }^{4}$ Department of Surgery, and McKusick-Nathans Institute for Genetic Medicine, Johns Hopkins University School of Medicine, 733N. Broadway, Baltimore, MD 21205, USA. Danhua Zhang and Keith P. Gates contributed equally to this work. Correspondence and requests for materials should be addressed to P.D.S.D. (email: ducdong@SBPdiscovery.org)
} 
n mammals and zebrafish, the hepatopancreatic ductal system is a network of tubular epithelium connecting hepatocytes of the liver and acinar cells of the pancreas to the intestine. Malformation and dysfunction of hepatopancreatic ducts can lead to pathologies including liver duct paucity and exocrine pancreas insufficiency-as found in patients with Alagille Syndrome (ALGS). ALGS is a congenital disease with a prevalence estimated at $1 / 70,000$ births, based neonatal liver disease ${ }^{1}$. It is associated with heterozygous mutations primarily in $J A G 1$, or less frequently in $\mathrm{NOTCH} 2^{2-5}$. A mechanistic understanding of the neonatal ductal defects in this disease will require rigorous investigation into the function of these genes during hepatopancreas duct development.

The duct lineage, defined by Sox 9 expression, has been proposed as a source of multipotent progenitors that contribute to the development, homeostasis, and regeneration of the liver and pancreas ${ }^{6}$. Subsequent studies on homeostasis and regeneration have both supported and disputed a role for duct cells as source for multipotent progenitors ${ }^{7-12}$. It is generally accepted that during early liver and pancreas development, bipotent (i.e., hepatoblasts) or multipotent cells give rise to both duct and hepatocytes or acinar cells. However, it remains unresolved whether specified duct cells during embryonic development also contribute to acinar and hepatocyte lineages ${ }^{6,13-15}$. Although Sox 9 is considered to be the earliest biliary marker ${ }^{16}$, lineage tracing Sox 9 expression might not be ideal because Sox 9 is not exclusively expressed in the duct lineage ${ }^{8,17}$. Using a more definitive duct lineage tracing CRE line and addressing the functional requirement of liver and pancreas duct cells will be necessary to resolve whether duct cells are a source of multipotent progenitors during organogenesis.

Although specific factors have been implicated in the lineage specification of the endocrine and acinar fates in the pancreas ${ }^{18,19}$, the genes required for induction of the entire ductal lineages in both the pancreas and liver (intrahepatopancreatic ducts, IHPD) have been elusive. Numerous studies have implicated Notch signaling in the morphogenesis and differentiation of both intrapancreatic ducts (IPDs) and intrahepatic ducts (IHDs) ${ }^{20,21}$. Ectopic expression of the Notch intracellular domain inhibits expression of hepatocyte and pancreatic acinar genes and enhances duct genes, supporting a role for Notch signaling in duct lineage specification ${ }^{22,23}$. However, the inability to completely and distinctly block the canonical Notch pathway in the pancreas and liver has confounded efforts to resolve whether this signaling pathway is specifically necessary for duct lineage induction, independent of its recognized requirement for differentiation, expansion, and maintenance of duct cells.

Given functional redundancy among Notch ligands and receptors, the predominant strategy to broadly block canonical Notch signaling has been to manipulate the more general components of the Notch pathway. However, down-regulation of canonical Notch activity by modulating the expression of Notch signaling components did not lead to complete loss of ducts, or yielded contrasting results. For example, while Maml1, Rbpj, Mib1, or Hes1 loss of function in the mouse pancreas can all lead to a reduction in duct lineage markers, the effects on the acinar and endocrine lineages differed $^{24,25}$. Further, loss of Hes1 and Rbpj also resulted in a broader pancreas hypoplasia phenotype. These differences may be due to varying levels of Notch loss of function or to non-Notch signaling specific effects, because none of the manipulated Notch signaling components are exclusively involved with canonical Notch signaling ${ }^{26-30}$. Furthermore, knockout of Notch receptor genes might also not be ideal because Notch receptors, independent of ligands, have been implicated in $B$-catenin signaling ${ }^{31}$.

More direct assessments of the role of Notch signaling in pancreas and liver duct specification may require analyzing the function of Notch ligands. Conditional loss of Jag1 from the mouse portal vein mesenchyme results in hepatic duct tube morphogenesis defects, leading to the current model suggesting that biliary paucity in ALGS arises via an analogous mechanismreduced JAG1 expression from non-endoderm derived cells causes biliary structural, not lineage specification, defects ${ }^{32}, 33$. The potentially incomplete efficiency of Cre/Lox based conditional knock out approaches, combined with the functional redundancy among Notch ligands, make it technically challenging to completely block Notch signaling in the mouse model at the ligand level. In support of redundant roles among Jagged ligands in liver and pancreas development, earlier studies using zebrafish showed that antisense morpholino (MO) knockdown of both jag $1 b$ and jag $2 b$ leads to more extensive disruption of pancreas and liver duct development than knockdown of either alone ${ }^{34}$. However, embryos with these factors knocked down continued to express the ductal marker Cytokeratin in what were described as hybrid hepatocytes and acinar cells. Whether early duct cells failed to be induced, appeared initially but failed to properly differentiate, or misdifferentiated into pancreatic endocrine cells, was not determined. Knockdown of either jag1b or $j a g 2 b$ alone can lead to an increase in the numbers of alpha-cells or beta-cells of the endocrine pancreas ${ }^{35}$. A decreased knockdown effectiveness at later stages is another possibility for the putative 'hybrid' cells. Together with concerns regarding off-target effects of morpholino ${ }^{36}$, more reliable loss of function models will be critical to rigorously assess the roles of Jag1b and Jag2b in development and disease.

To investigate the effect of specific, consistent, and complete loss of Jag1b and Jag2b function on liver and pancreas development, we analyzed zebrafish jag $1 b$ and jag $2 b$ mutants, using early and late organ cell lineage markers, as well as a transgenic reporter of canonical Notch signaling activity. Our studies yield evidence that Jag $1 \mathrm{~b}$ and Jag $2 \mathrm{~b}$ are the primary ligands required for induction of all detectable canonical Notch signaling and all duct cells within the developing liver and exocrine pancreas and that duct cells are not a significant source of progenitors for the hepatocyte or pancreas acinar lineages. Further, stage specific loss of function studies suggest that, distinct from its later role in preventing pancreatic duct cells from adopting endocrine fate, Notch signaling is required during early pancreogenesis to establish the ductal compartment. Our chimera studies demonstrate that Jagged ligands from endoderm-derived cells within the liver and pancreas are able to directly induce Notch signaling and the duct lineage, a mechanism that contrasts with how Jag1 signaling is thought to regulate duct development in mammals and in ALGS.

\section{Results}

Liver and exocrine pancreas Notch activity requires Jagged. In zebrafish, both jag $1 b$ and jag $2 b$ are widely expressed in the hepatopancreatic domain during development (Supplementary Fig. 1). To assess the role of these ligands using a more consistent and reliable loss of function approach, we examined jag $1 b^{b 1105}$ and jag $2 b^{h u 3425}$ mutants. Both mutants carry nonsense mutations within the N-terminal coding region of their respective genes, and thus are likely to be complete loss-of-function mutants. These mutations do not lead to lethality until juvenile stages, allowing for analysis of Jagged loss-of-function in the developing liver and pancreas. To examine canonical Notch activity in the pancreas and liver, these mutants 37,38 were analyzed with a Tp1:GFP transgenic reporter. The Tp1:GFP reporter, which has GFP driven by a regulatory element containing $12 \mathrm{RBPJk}$ binding sites from the promoter of Terminal Protein 1, has been demonstrated to effectively mark cells with canonical Notch signaling activity ${ }^{39}$. 
With this line, we confirmed that intrapancreatic and intrahepatic ducts, but not the extra hepatopancreatic ducts, have high canonical Notch activity, based on GFP co-expression with AnnexinA4 (Anxa4), which is broadly expressed in fish and mammalian hepatopancreatic ducts ${ }^{40,} 41$. In the exocrine pancreas, all Tp1:GFP expressing cells are also positive for Nkx6.1 (Supplementary Fig. 2a), a transcription factor also expressed in the ductal compartment of the mouse pancreas ${ }^{42}$. Within the
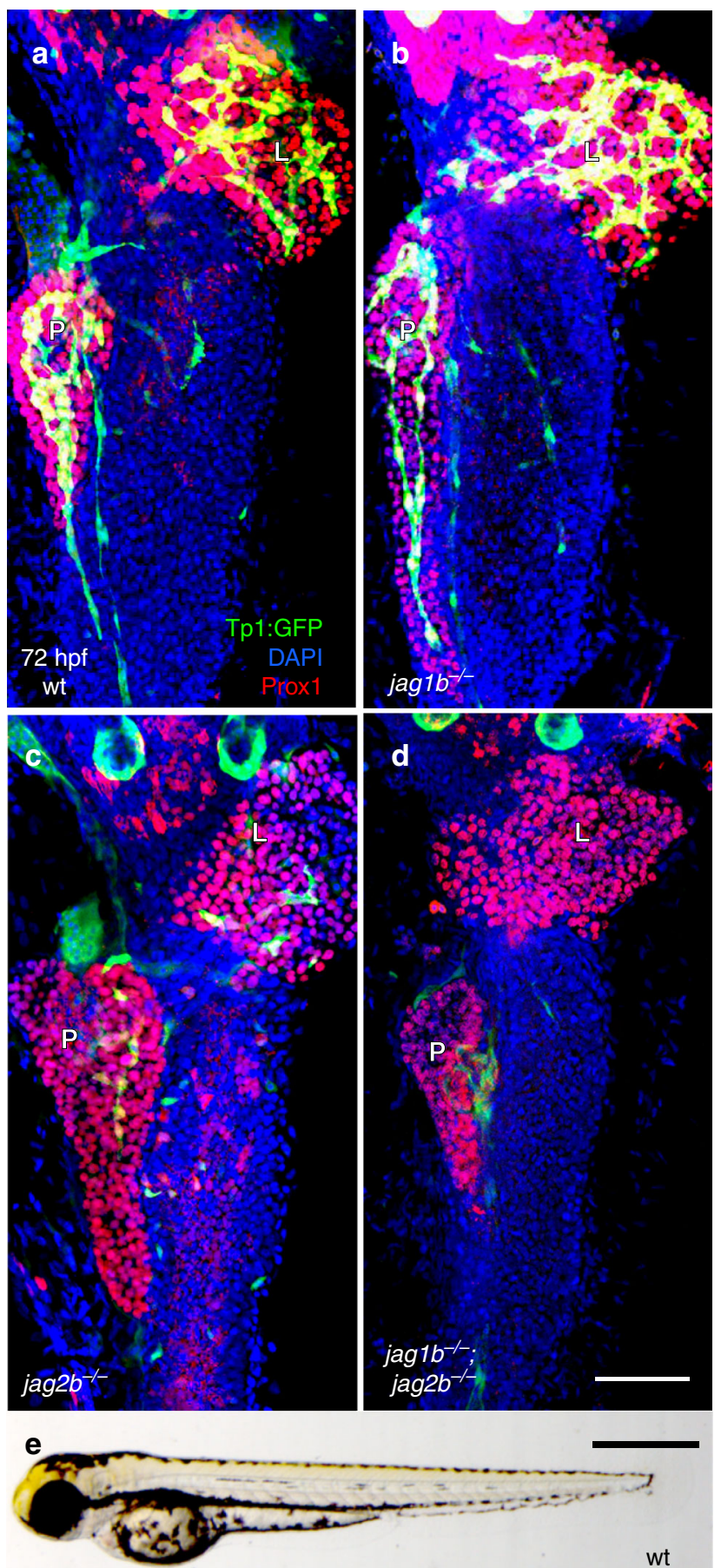

wt

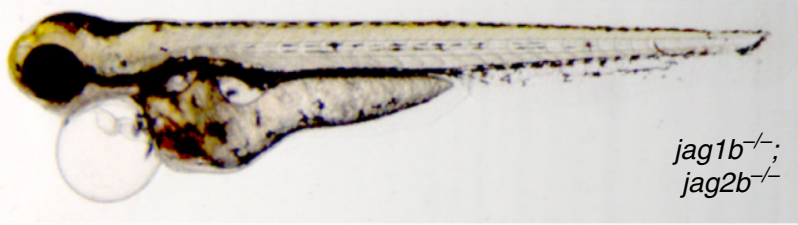

developing liver, all cells expressing Prox1 (a broad marker of liver and exocrine pancreas endoderm), that lack the hepatocyte early marker $\mathrm{Hnf}_{4} \mathrm{a}^{43}$, are Tpl:GFP positive (Supplementary Fig. 2b), indicating that all endoderm-derived cells are either Hnf4a positive or Tp1:GFP positive. These findings demonstrate that within the liver and exocrine pancreas, Notch activity, based on the Tp1 promoter, is strictly limited to and labels all duct cells. Tp1:GFP positive cells that are negative for Proxl can also be found in and around the liver and pancreas, indicating the presence of non-endoderm, Notch active tissues. Examination of Tp1:GFP expression in conjunction with an endothelial reporter $k d r l: m$ Cherry $^{44}$, demonstrates that these Notch active cells are indeed endothelial cells (Supplementary Fig. 2c, d). Tp1:GFP expression is also observed in a subset of pancreatic Islet1 expressing endocrine cells (Supplementary Fig. 2d).

Homozygous jag1 $1 b^{b 1105}$ mutant 72 hours post fertilization (hpf) embryos $(n>15)$ show no obvious decrease in Tp1:GFP expression in both the liver and pancreas (Fig. 1b). However, jag $2 b^{\text {hu}} 3425$ homozygous mutants $(n=26)$ do exhibit a reduction in the number of Tp1:GFP expressing cells. 77\% of jag $2 b^{h u 3425}$ homozygotes have a partial loss of Tp1:GFP ${ }^{+}$cells in both the liver and exocrine pancreas (Fig. 1c). The remaining embryos have 2, 1, or no Tp1:GFP expressing cells in the entire liver and exocrine pancreas (not shown), suggesting that Jag2b is the primary Notch ligand required for most Notch signaling during liver and pancreas development. The variable penetrance may be indicative of functional redundancy with other Notch ligands, as previously demonstrated by the more severe ductal defects in $j a g 1 b$ and jag2 $b$ knockdown studies ${ }^{34}$. However, Notch activity was not assessed in that knockdown study. We find that double homozygous embryos (jag1b ${ }^{-/} ; j a g 2 b^{--}$) can survive past $72 \mathrm{hpf}$, when differentiation markers for pancreas acinar cells and hepatocytes are prominent. Bright field microscopy of double mutants indicates heart and pronephric edema to be the most obvious defects, with no apparent developmental delay (Fig. 1e). We find that all jag1b ${ }^{-/}$;jag $2 b^{-/-}$embryos $(n=15$ Fig. 1d) lack Tp1:GFP expression in the entire Prox $1^{+}$exocrine pancreas, while GFP can still be detected in the endocrine pancreas and the vasculature associated with the pancreas (Fig. 1a, Supplementary Fig. 3c). Loss of Notch activity is also observed in the Prox $1^{+}$liver domain, where approximately $33 \%$ of $j a g 1 b^{-/-} ; j a g 2 b^{-/-}$mutants also completely lack Tp1:GFP cells, while the remaining have only 1 or $2 \mathrm{Tp1}: \mathrm{GFP}^{+}$cells (Supplementary Fig. $4 \mathrm{a}, \mathrm{b} n=15$ ). These results indicate that Jag1b and Jag2b can be responsible for all detectable canonical Notch activity in the exocrine pancreas and liver. With a complete lack of canonical Notch signaling in jag $1 b^{-/-} ; j a g 2 b^{-/-}$mutants, we have a more definitive model to investigate the specific role of ligand-induced canonical Notch signaling in liver and pancreas development.

Fig. 1 Jag $1 b$ and Jag $2 b$ are required for all canonical Notch signaling in the developing liver and exocrine pancreas. a-d 3D rendering of the foregut endoderm of $72 \mathrm{hpf}$ embryos in the canonical Notch signaling transgenic reporter $\operatorname{tg}(\mathrm{Tp1}: \mathrm{GFP}$ ) (green) background, stained with hepatopancreatic endoderm marker Prox1 antibodies (red) and nuclear marker DAPI (blue). Ventral view, anterior up. Tp1:GFP expression in liver and pancreas of jag1b $-/-$ mutants (b, representative sample, $n>15$ ) is comparable to that in wild type (a), but is decreased in jag $2 b^{-/-}$mutants (c, representative of 14/ 18 samples). jag $1 b^{-/-} ; j a g 2 b^{-/-}$double mutants (d, representative sample, $n=15)$ show a complete absence of Tp1:GFP expression in Prox $1^{+}$cells. e Bright-field microscopy of $4 \mathrm{dpf}$ wild type (top) and jag $1 b^{-/-} ; j a g 2 b^{-/-}$ mutant (bottom), showing mutant with grossly normal development with the exception of heart and pronephric edema. Samples from three different jag $1 b^{-/+} ; j a g 2 b^{-/+}$in-cross clutches. Scale bars $50 \mu \mathrm{M}$ (a-d), and $500 \mu \mathrm{M}$ e 
Intrahepatopancreas duct lineage specification requires Jagged. To investigate the effect of canonical Notch signaling loss on the entire hepatopancreatic ductal system, we analyzed early markers distinguishing ducts from other pancreas and liver cell types. One of the earliest distinguishing features of the liver duct lineage is the lack of expression of the hepatocyte marker Hnf4a in Prox1 positive cells. As the liver matures, the hepatic duct cells maintain a high level of Alcama expression, whereas hepatocytes exhibit
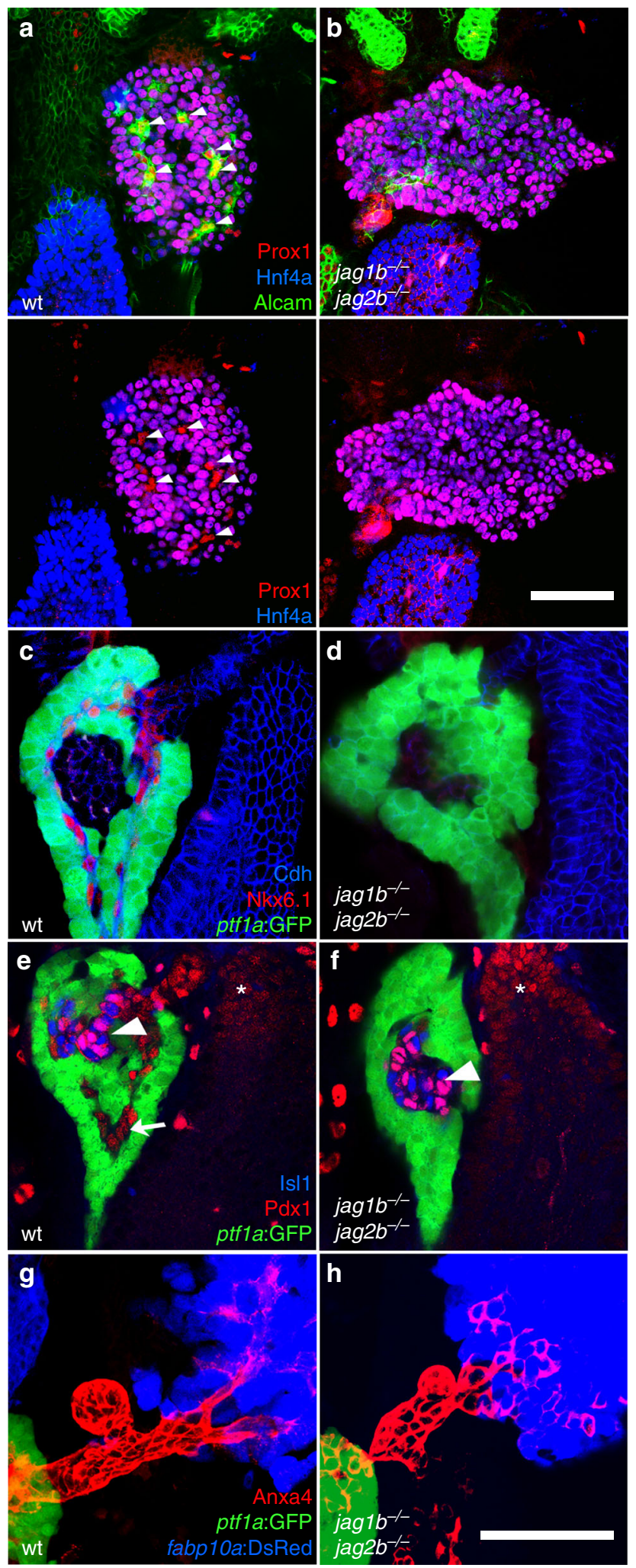

reduced or absent Alcama expression (Fig. 2a, Supplementary Fig. 4c). Whole-organ confocal microscopy revealed jaglb jag $2 b^{-1-}$ livers with Hnf4a expressed in all Prox $1^{+}$cells, suggesting a complete lack of duct lineage. Consistently, highlevel Alcama expression was almost completely lost (Fig. 2b), with the remaining expression associated with Prox $1^{+} / \mathrm{Hnf}_{4} \mathrm{a}^{+}$cells (Supplementary Fig. 4d). Cleaved Caspase3, an apoptosis marker, was not observed in the double mutant liver (Supplementary Fig. $5 \mathrm{a}-\mathrm{d}$ ) or pancreas (not shown), suggesting the lack of duct cells is not due to cell death. These results indicate that Jaglb and Jag2b are essential for intrahepatic duct lineage specification.

During early ventral pancreas development in both mouse and zebrafish, $p t f 1 a$ is initially co-expressed with $n k x 6.1$ and $p d x 1$. As the duct and acinar lineages of the exocrine pancreas become specified, ptf1a expression becomes restricted to acinar cells while $n k x 6.1$ and $p d x 1$ become restricted to duct cells ${ }^{45,46}$. By $72 \mathrm{hpf}$, $n k x 6.1$ and $p d x 1$ are only expressed in duct cells that have very low or undetectable ptf1a expression. In jag $1 b^{-/-} ; j a g 2 b^{-/-}$embryos at $72 \mathrm{hpf}, \mathrm{Nkx} 6.1$ and Pdx1 are undetectable in the exocrine pancreas of all double mutants, whereas the pattern of ptf1a:GFP expression appears more contiguous throughout the exocrine pancreas (Fig. 2d, f). However, Pdx1 expression in the Islet $1^{+}$endocrine pancreas is not lost in the double mutants, indicating that Pdx1 loss is specific to the exocrine pancreas. These results indicate that Jag $1 b$ and Jag $2 b$ are essential for the lineage specification of the intrapancreatic duct. In jag $1 b^{-/-} ;$jag $2 b^{-/-}$embryos, expression of Anxa4 in extrahepatopancreatic ducts ${ }^{41,} 47$ remains (EHPD; Fig. $2 \mathrm{~h}$ ), while the elevated expression normally observed in the IHPD cells is lost. This finding indicates that the EHPD can still form in jag1b $1 b^{-/} ;$jag $2 b^{-/-}$embryos, whereas the duct cells within the liver or pancreas are absent. This regionalized defect is consistent with detection of Notch activity in the IHPDs but not the EHPDs.

Early and late roles of Notch in duct and endocrine lineages. Different models of Notch inhibition can result in a loss of duct cells that is associated with either excessive or reduced endocrine cell differentiation ${ }^{20,39,48,49}$. In the $j a g 1 b^{-/-} ; j a g 2 b^{-/-}$mutants, all pancreatic endocrine cells (Islet1 ${ }^{+}$or neuroD:GFP ${ }^{+}$) were found closely associated with the principal islet $(100 \% n=18$, Fig. 3a, b), suggesting the absence of endocrine neogenesis. Consistently, the total number of pancreatic endocrine cells in the double mutants $\left(87( \pm 6)\right.$ Islet $^{+}$cells, $\left.n=8\right)$ is significantly lower than in wild type at $89 \mathrm{hpf}\left(113( \pm 23)\right.$ Islet $^{+}$cells, $\left.n=10\right)$. Our analysis does not show a consistent change in the size of the acinar pancreas in double mutants (see Figs. 2c-f and 3a,b).

Fig. 2 Jag $1 b$ and Jag2b are required for intrahepatopancreatic duct lineage specification. $\mathbf{a}-\mathbf{h}$ Whole organ immunofluorescence expression analysis of the $72 \mathrm{hpf}$ foregut endoderm of wild type and jag $1 b^{-/-} ; j a g 2 b^{-/-}$mutant embryos. (a, b Alcam channel removed in bottom panels). Intrahepatic duct cells marked by high Alcama levels (a, green), and by Prox1 (red) but not Hnf4a expression (blue, a), are found in wild type liver (arrowheads, a). These duct cells are not found in the jag $1 b^{-/-} ; j a g 2 b^{-/-}$mutant liver (b, representative sample, $n=13$ ), which is comprised entirely of hepatocytes expressing both Prox1 and Hnf4a. c-f Intrapancreatic ductal cells expressing $\mathrm{Nkx6.1}$ (red, c, d) or $\mathrm{Pdx} 1$ (red, arrow, e, f) are present in wild type but not in jag1 $b^{-/-} ;$jag $2 b^{-/-}$mutants. Pdx1 expression in Islet $1^{+}$ cells (blue, arrowheads) and the duodenum (asterisk) is comparable to wildtype siblings, as is expression of ptfla:GFP+ (green) acinar cells c-f and $\mathrm{Anxa4}^{+}$(red) extrahepatopancreatic duct cells (red, g, h). g, h Anxa4 ${ }^{+}$(red) extrahepatopancreatic ducts can be found joining the ptfla:GFP ${ }^{+}$(green) pancreatic acinar cells to the fabp10a:DsRed ${ }^{+}$hepatocytes (blue). Representative samples from three different $j a g 1 b^{-/+} ; j a g 2 b^{-/+}$in-cross clutches. Scale bars, $50 \mu \mathrm{M}$ 


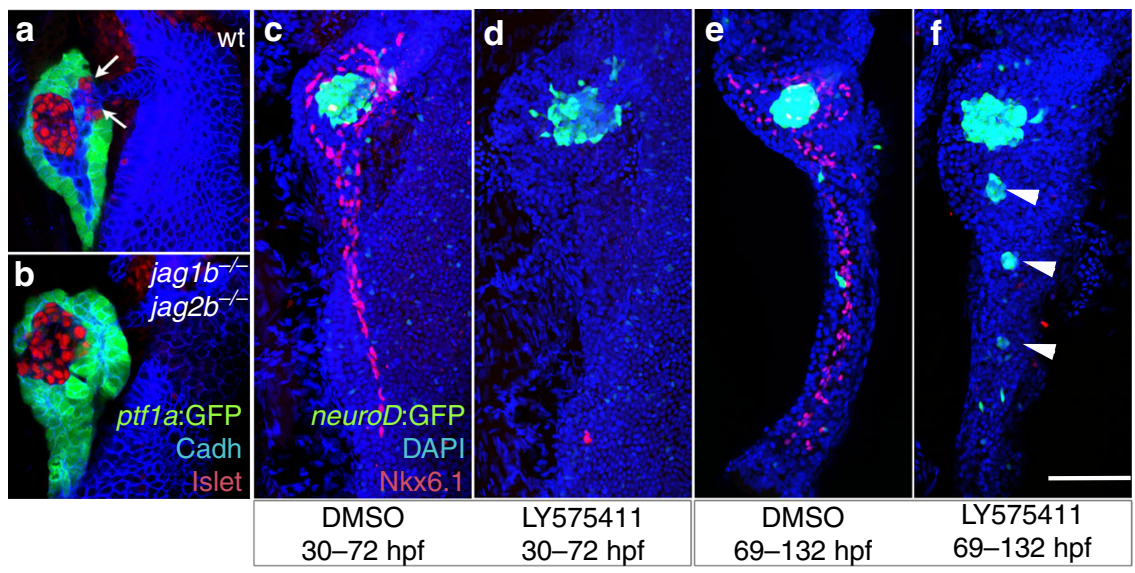

Fig. 3 Distinct early and late roles of Notch signaling in regulating duct and endocrine lineages. a, b Pancreas of 60 hpf jaglb embryos. Neogenic endocrine cells expressing Islet1 (red) are normally found outside the principal islet at the base of the wild type pancreas (arrows, a). These neogenic endocrine cells are not present in the $60 \mathrm{hpf} j a g 1 b^{-/-} ; j a g 2 b^{-/-}$mutant pancreas (b, representative sample, $n=18$, pooled from three different jag $1 b^{-/+} ; j a g 2 b^{-/+}$in-cross clutches), which is entirely ptfla:GFP (green) with the exception of the principal islet. c, $\mathbf{d}$ Pancreas of 5 dpf-treated embryos. Early inhibition of Notch signaling with gamma secretase inhibitor LY575411 treatment (d, $n=9$ and $\mathbf{c}, n=5$. Samples from 2 different experiments) at 30-72 hpf leads to loss of Nkx6.1 $1^{+}$pancreatic duct (red) without excess neuroD:GFP ( treatment ( $\mathbf{f}, n=9$, and $n=6$ for control, e Samples pooled from 2 different experiments) also leads to loss of nkx6. $1^{+}$pancreatic duct (red) that is coupled with excess neuroD:GFP ${ }^{+}$(green) endocrine cells (arrowheads, $\mathbf{f}$ ). Scale bar, $50 \mu \mathrm{M}$

To formally test whether the contrasting endocrine phenotypes among different Notch loss-of-function models reflect temporally distinct functions of Notch signaling during pancreas development, we blocked Notch signaling at different stages of pancreas development using a gamma secretase inhibitor (GSI), LY $411575^{50}$. While the function of gamma secretase has been implicated in multiple signaling pathways ${ }^{51}$, chemical inhibitors of gamma secretase, including LY411575, has become an accepted approach to effectively block the Notch pathway in multiple animal models and organ systems. Importantly, we find that

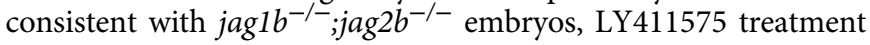
starting prior to specification of the ventral pancreas leads to a total absence of Nkx6.1 positive pancreas duct cells at 5 days post fertilization (dpf) (Fig. 3d). All neuroD:GFP ${ }^{+}$pancreas endocrine cells were found closely localized to the principal islet and not in the tail of the pancreas, suggesting a lack of excessive endocrine mis-differentiation. Although later treatment with LY411575 also leads to a loss of Nkx6.1 expressing duct cells, this defect is coincident with excessive neuroD:GFP ${ }^{+}$endocrine cells in the tail of the pancreas (Fig. 3f). Together, our findings support a model whereby Notch signaling function is initially required for pancreatic duct lineage specification and indirectly for neogenic endocrine lineages, but is later required for maintenance of the ductal lineage via repression of endocrine fate. This model reconciles the contrasting data on the effect of Notch loss-offunction during pancreas endocrine and duct development. However, the possibility of the GSI treatment affecting other genetic pathways cannot be excluded. Further, in addition to temporal distinct roles for Notch signaling, spatial and ligands/ receptors specific functions may also contribute to pancreas and liver development.

Acinar pancreas and hepatocyte lineages do not require IHPD cells. jag $1 b^{-/-} ; j a g 2 b^{-/-}$embryos provide a unique opportunity to examine the consequence of complete absence of canonical Notch signaling and duct cells during liver and pancreas organogenesis. Despite the mechanical pressure on the developing liver and pancreas by the adjacent heart and kidney edema found in these embryos, some double mutants do have livers and pancreata that are relatively normal in size and shape (Figs. 1-3). At $5 \mathrm{dpf}$, similar to the wild type pancreas, the double mutant acinar cells are polarized, as indicated by apical localization of ZO-1 (Figs. $4 a, b$ ) and zymogen granules. The formation of acinar lumens is obvious and can appear cystic in the double-mutant pancreas despite a complete lack of duct cells, providing evidence that duct cells are non-essential for exocrine pancreas lumen formation. In the jag1 $1 b^{-/-} ; j a g 2 b^{-/-}$liver, ZO-1 is still detectable at the apical side of hepatocytes where lumens are apparent, despite also lacking duct cells (Figs. 4c, d). fabp10a:DsRed reporter ${ }^{47}$ expression can be found in all Prox1-expressing cells in the double mutant liver at $5 \mathrm{dpf}$, demonstrating broad hepatocyte differentiation (Fig. 4d). Together, these results lend functional evidence suggesting that intrahepatopancreatic ducts are not essential for the development of acinar and hepatocyte compartments of these organs.

Our discovery that Jagged/Notch signaling is the induction event for IHPD lineage specification suggests that tracking Notch active cells may be most reliable for assessing lineage contribution from duct cells. Further, the appearance of Tp1:GFP prior to the resolution of Nkx6.1, Pdx1, and Prox1 expression to the pancreatic ducts implicates Notch activity as the earliest known specific marker of the duct lineage. To determine whether Notch active cells contribute to the developing acinar and hepatocyte lineages, we lineage traced canonical Notch active cells using a transgenic line with tamoxifen inducible Cre driven by the Tp1 promoter $\left(\mathrm{Tp} 1: \mathrm{CreER}{ }^{\mathrm{T} 2}\right)^{52}$, in combination with a Cre reporter line, ubb:lox-CFP-STOP-lox-H2b-mCherry (Fig. 4e). Previous zebrafish pancreas development studies found a few Tp1: $\mathrm{CreER}^{\mathrm{T} 2+}$ cells contributing to the ptfla: $\mathrm{GFP}^{+}$acinar compartment with tamoxifen added at either 12 or $56 \mathrm{hpf}^{53}$. To initiate lineage tracing of $\mathrm{Tp}^{+}$cells at the earliest stages of liver and pancreas development, we induced Tp1:CreER ${ }^{\mathrm{T} 2}$ activity starting at $30 \mathrm{hpf} \mathrm{H} 2 \mathrm{~B}-\mathrm{mCherry}$ marked an average of $79 \%$ of Prox $1^{+}$, Hnf4a-negative duct cells (Fig. 4g; total 1,416 cells, 12 embryos) in the liver and $75 \%$ of Nkx6.1 $1^{+}$and ptf1a:GFP or Elastase negative duct cells (Fig. 4f; total 1,586 cells, 24 embryos) in the pancreas. In some individual samples, over $90 \%$ of the intrahepatic ducts were labeled, demonstrating the effectiveness of this lineage tracing approach. We observed no labeling of hepatocytes $\left(\mathrm{Hnf}_{4} \mathrm{a}^{+}\right)$and very few of acinar cells (ptfla:GFP ${ }^{+}$or elastase $^{+}$, and Nkx6.1 negative). Of 24 embryos, 20 had no 
labeling of acinar cells, and 4 had a total of only 12 acinar cells labeled, versus the 2,578 labeled intrapancreatic duct cells in these same embryos. Collectively, our lineage tracing studies suggest that Notch active cells do not contribute to the hepatocyte lineage and potentially contribute very few cells to the acinar lineage during organogenesis.
Endoderm Jagged induces Notch activity and duct lineage. Conditional inactivation of Jag1 from the portal vein mesenchyme led to liver duct tubulogenesis defects, but did not prevent the expression of Hes1, a marker of Notch signaling, or early liver duct markers, suggesting that Jag1 expression from the liver vasculature is not essential for liver Notch signaling or duct
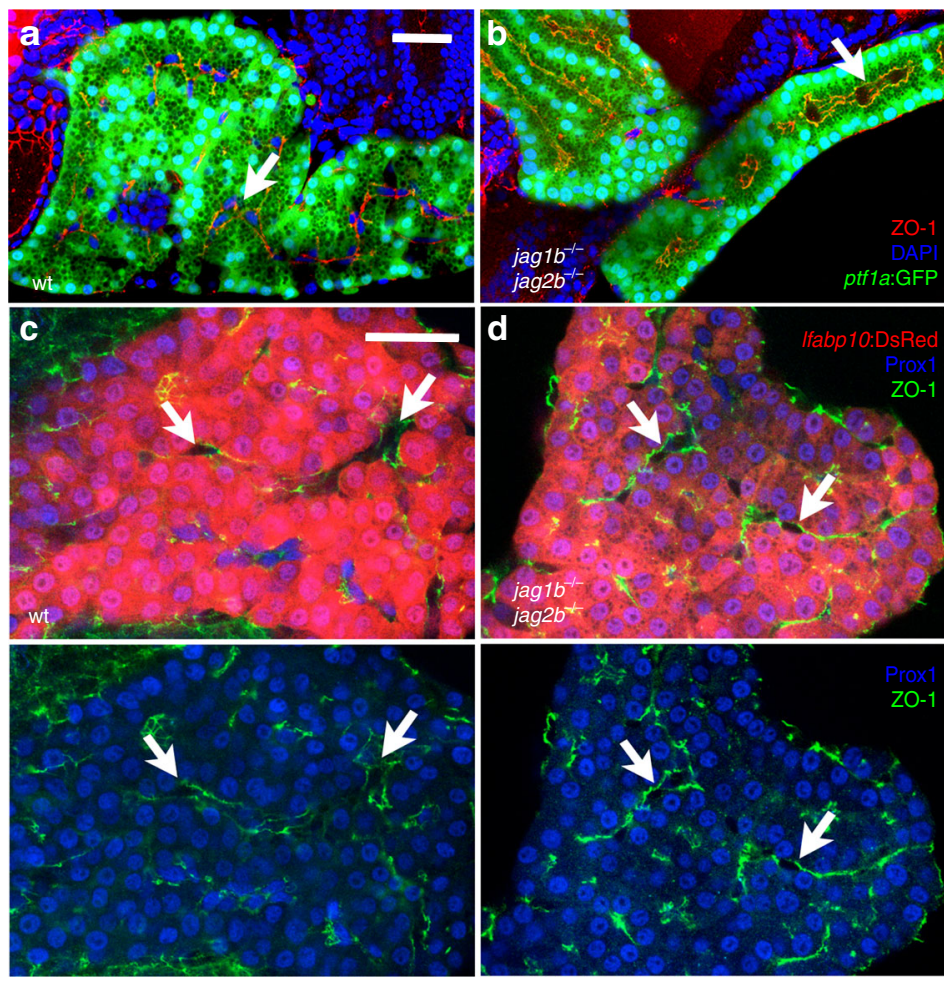

e
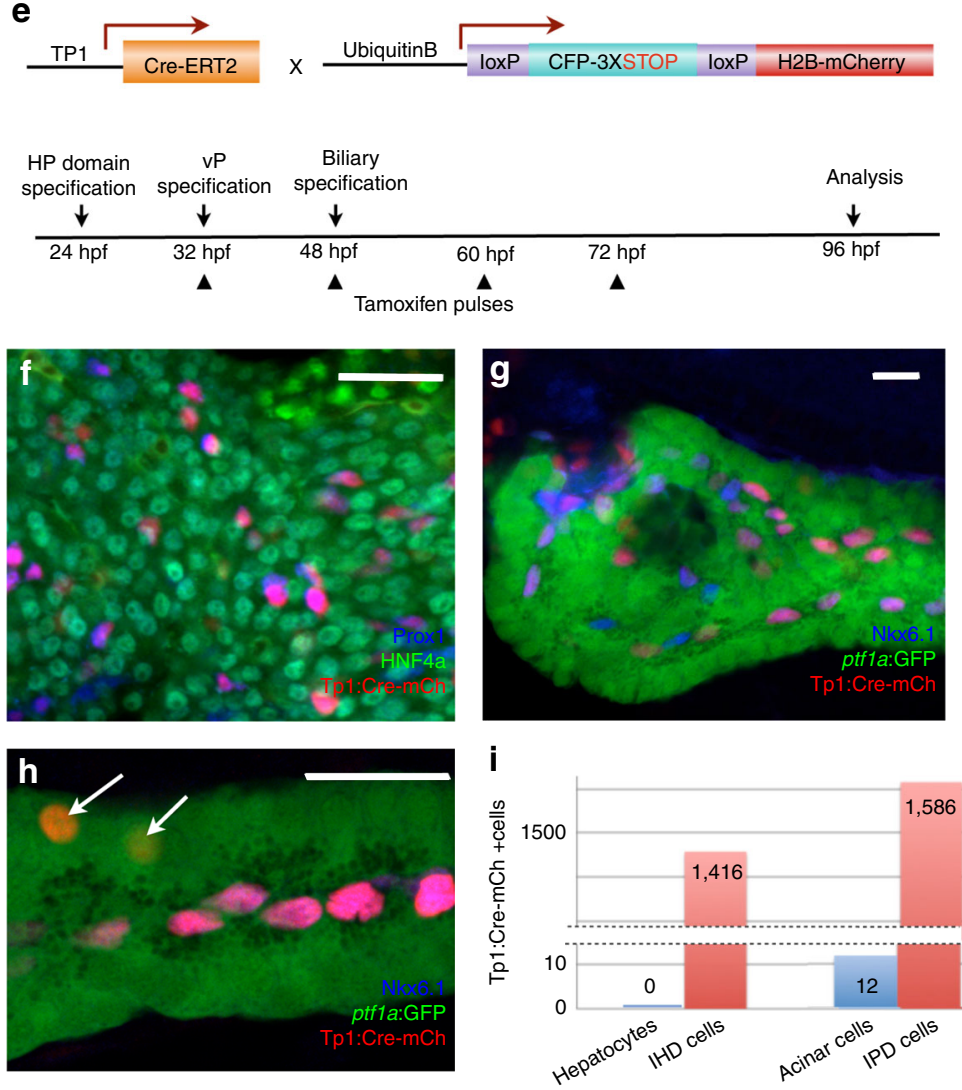
lineage specification ${ }^{33}$. Consistently, liver duct specification is also not lost in zebrafish npasl4 mutants, which fail to develop the vasculature (Supplementary Fig. 6) ${ }^{34,54,55}$. It is not known whether endoderm sources of Jagged ligands are required to directly activate Notch signaling and induce IHPD specification. Jag1 was shown to be expressed endoderm-derived cells in the embryonic mouse liver and pancreas, and conditional loss of Jag1 from the pancreatic endoderm using Pdx1-Cre leads to a postnatal reduction and malformation of pancreatic ducts $^{33}, 48,56$. These findings suggest that Notch signaling in these organs may be directly induced by Jagged signaling from adjacent endoderm-derived cells. To functionally test whether normal Jag expression in endoderm-derived cells of the liver and pancreas is sufficient for Notch activation and duct lineage specification, we analyzed embryos chimeric for jagged expression in the endoderm using a standard transplantation approach. We injected sox32 mRNA to induce endoderm fate and a rhodamine dextran dye to fluorescently label the entire donor embryo. These labeled endoderm donor cells were then transplanted into unlabeled host embryos with jag1b and jag $2 b$ knocked down by morpholinos $^{57}$ (Fig. 5a). The use of mutants was impractical for these transplantation experiments, as only $1 / 16$ offspring are expected to be double homozygous for jag $1 b$ and jag $2 b$ from a double heterozygote incross. To assess canonical Notch activity, Tp1:GFP was used for both donor and host embryos. In embryos in which the pancreas and liver are chimeric $(n=10)$, there are no Tp1:GFP expressing cells found in regions of these organs that lack rhodamine dextran labeled wild type donor cells. However, Tp1:GFP ${ }^{+}$cells were only found in regions of the organs with rhodamine dextran labeled wild type donor cells. Importantly, Tp1:GFP is observed in both donor (rhodamine $^{+}$) and morphant host cells (rhodamine-) that are directly associated with the wild type donor cells (Figs. 5b, c). These Tp1:GFP cells are Nkx6.1 $1^{+}$in the pancreas and express high levels of Alcama in the liver, suggesting that they are indeed ductal cells. These findings suggest that Jagged ligands from endoderm derived cells in the liver and pancreas can directly induce Notch activity and IHPD lineage specification in adjacent cells.

\section{Discussion}

The investigation presented here addresses several fundamental outstanding questions regarding intrahepatopancreatic duct specification, function, and disease. The unique ability to generate jag $1 b / 2 b$ compound homozygous embryos that are viable throughout organogenesis was critical for these studies. Our mutant analysis demonstrated that just two ligands, Jaglb and Jag2b, are responsible for all detectable canonical Notch signaling and duct lineage specification in both the exocrine pancreas and liver of zebrafish (summarized in Fig. 6). Notch signaling has been linked to cell cycle due to the pancreatic hypoplasia observed with loss of Notch associated factors Rbpjk, Hes1, and Sox $9^{58}$. However, the lack of any detectable canonical Notch signaling and the normal pancreas size in $j a g 1 b^{-/-} ; j a g 2 b^{-/-}$ embryos suggest that the function of these nuclear factors in early pancreas growth is not associated with ligand-dependent canonical Notch signaling. jag1 $b^{-/-} ; j a g 2 b^{-/-}$embryos also helped distinguish the roles of Notch signaling in duct lineage specification versus maintenance. Loss of pancreatic duct cells in these double mutants is not coupled with excessive endocrine differentiation as observed with other Notch loss-of-function approaches. Differences in stage and severity of Notch signaling loss may explain the phenotypic differences found. We conclude that during pancreas development, Notch signaling function is initially required for duct lineage specification and indirectly for neogenic endocrine lineages, and is later required for repression endocrine fate to maintain the ductal lineage (summarized in Fig. 6).

With a complete lack of duct lineage specification in double mutants, it was surprising to find that the developmental specification and morphogenesis of the acinar and hepatocyte compartments were not overtly affected. This finding was especially unexpected because the IHPDs are thought to play a central role in organogenesis, contributing progenitors to the development of the acinar and hepatocyte compartments ${ }^{6,13-15}$. Our studies provide functional evidence using jagged mutants and more definitive lineage tracing data using the earliest duct specific reporter to reveal that ducts of the liver and pancreas are not essential for and do not contribute significant numbers of cells to the developing hepatocyte and acinar lineages. A profound understanding of liver and pancreas progenitor biology will shape our perspectives on regeneration and diseases associated with these organs such as cancer, diabetes, and Alagille syndrome.

In the complete absence of Jagged/Notch signaling in double mutant embryos, we posit that cells that would normally contribute to intra hepatopancreatic ducts are mis-specified to adopt acinar and hepatocyte fates. Because Jagged expressing cells function to induce Notch signaling and the duct lineage, and no duct specific genes are activated in these mutants, formal lineage tracing experiments to determine the lineage derivatives of presumptive ducts are currently not possible. However, it has been shown that ectopic Notch signaling by misexpression of the Notch intracellular domain can induce duct lineage markers in acinar cells and hepatocytes, suggesting that Notch signaling can promote ductal fates at the expense of hepatocyte and acinar fates 22,23 . Together with our loss of function studies, we favor a model in which Notch functions during early pancreas and liver development to redirect acinar and hepatocyte precursors towards adopting duct fate instead (summarized in Fig. 6). This model argues that acinar and hepatocyte lineages are default in the absence of Notch signaling.

Based on mouse studies of Jag1 function, the prevailing model suggests that liver duct paucity in ALGS is caused by liver duct tubulogenesis defects that are due to insufficient JAG1 signals from non-endoderm derived cells ${ }^{33}$. However, the findings

Fig. 4 Intrahepatopancreatic ducts do not contribute significantly to the developing acinar and hepatocyte lineages. a-d Immunofluorescence analysis of

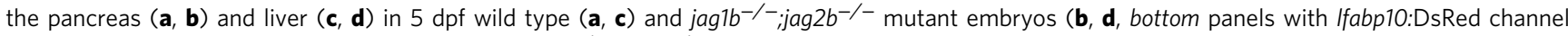
removed). Despite the absence of ductal cells in jag $1 b^{-/-} ; j a g 2 b^{-/-}$mutants $\mathbf{b}$, d, lumen formation (arrows) and apical localization of ZO-1 (red in $\mathbf{b}$, green in $\mathbf{d})$ remain comparable to wild type siblings $(\mathbf{a}, \mathbf{c})$. Normal differentiation indicated by zymogen granule accumulation in the ptf1a:GFP ${ }^{+}$pancreatic acinar cells (green) and Ifabp-DsRed expression (red) in hepatocytes. e Schematic of approach to Notch activity lineage tracing. $\mathbf{f}$-i Notch active labeled $\mathrm{H}_{2} \mathrm{~B}-\mathrm{mCherry}{ }^{+}$cells (red) in the liver are all Prox $1^{+}$(blue) and $\mathrm{Hnf}_{4} \mathrm{a}^{-}$(green) ( $n=12$ embryos, 1,416 labeled cells counted from two different experiments (f), $P<0.0001)$. g, h In the pancreas, nearly all H2B-mCherry ${ }^{+}$cells (red) co-express Nkx6.1 (blue) and are ptf1a:GFP ${ }^{-}$(green) ( $n=24$ embryos, 1,586 labeled cells counted from 3 different experiments, $P<0.0001) \mathbf{g}$ Only a few H2B-mCherry ${ }^{+}$cells that lack Nkx6.1 but are ptf1a:GFP ${ }^{+}$were observed (arrows, h. $n=4 / 24$ embryos, 12 cells total). $\mathbf{i}$ Graph summarizing numbers of Notch active labeled H2B-mCherry ${ }^{+}$cells found in hepatocytes versus intrahepatic duct (IHD) cells and acinar pancreas versus intrapancreatic duct (IPD) cells. Representative samples; a-d, $n=4$ each. Scale bars $25 \mu M$ 

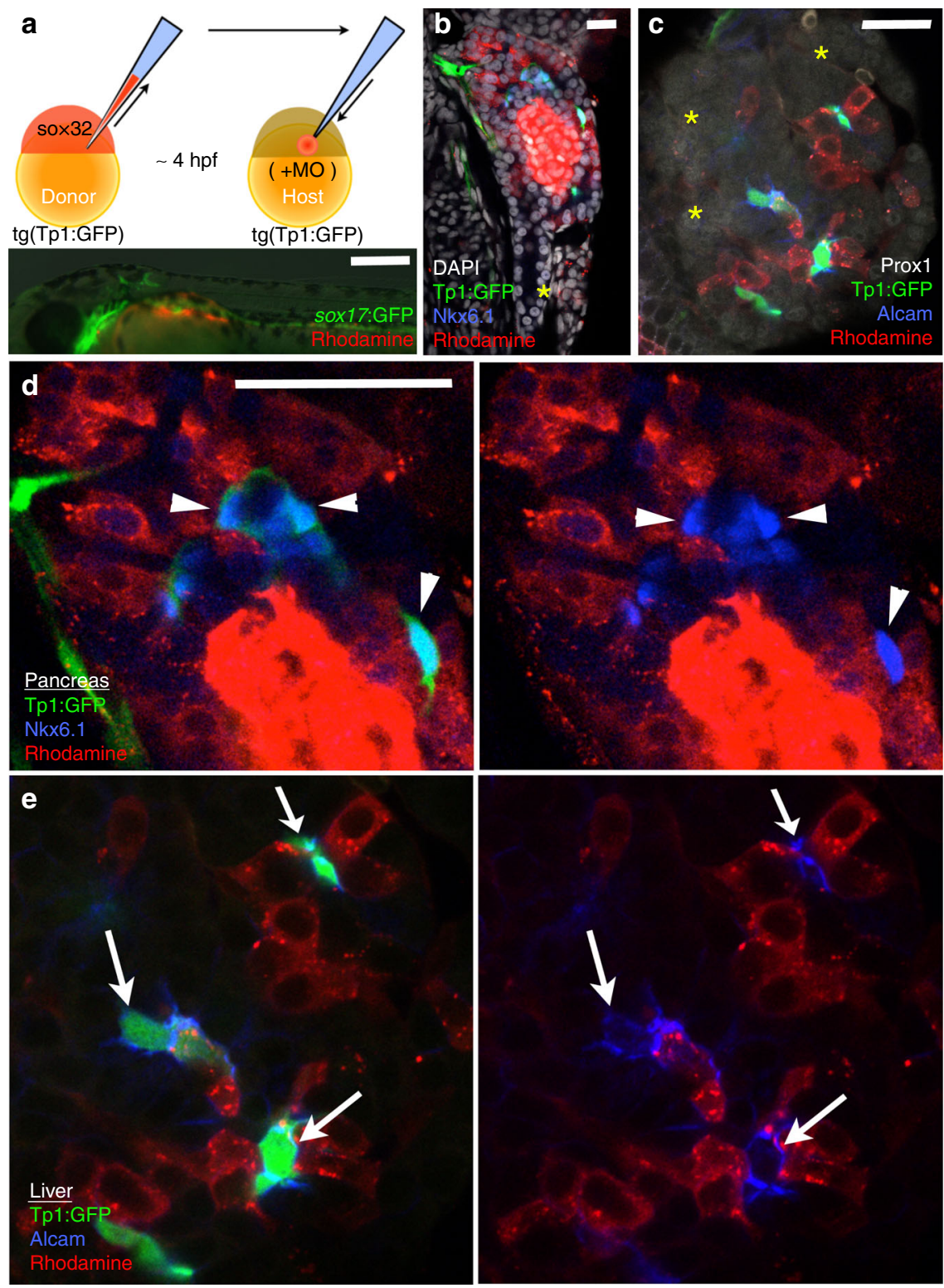

Fig. 5 Endoderm expression of Jagged induces Notch activity and lineage specification. a Schematic of transplant approach to generate endoderm chimeras. At the one-cell stage, wild type Tp1:GFP donor embryos were injected with rhodamine dextran for lineage tracing and sox32 mRNA for conversion to endoderm. At late blastula stage, donor cells were transplanted into Tp1:GFP host embryos that had been injected with antisense MO's to knockdown Jag1a and Jag1b. (a, bottom panel) Example of endoderm chimeric embryo demonstrating rhodamine dextran labeled donor cells (red) localized primarily to the sox 17:GFP ${ }^{+}$(green) host gut endoderm region. b, c Immunofluorescence analysis of chimeric host pancreas (b) and liver (c) generated by the approach described in a demonstrating a rescue of Notch signaling in Jag1b/Jag2b compromised embryos by endoderm cells with wild type expression of Jag1b/Jag2b. Tp1:GFP ${ }^{+}$cells (green) are only observed in regions of these organs with wild type donor cells (red) but not in regions without donor cells (yellow asterisks), (d, e) (representative samples, $n=10$ embryos. Samples pooled from 2 experiments). Rescued Tp1:GFP ${ }^{+}$cells found without rhodamine dextran (red, white arrows and arrowhead), are always directly associated with rhodamine dextran + cells. All rescued Tp1:GFP + host cells are Nkx6.1 ${ }^{+}$(blue) in the pancreas (d) and Alcama ${ }^{+}$(blue) in the liver (e), indicating that they are of the duct lineage. Scale bars $250 \mu \mathrm{M} \mathbf{a}$, and $25 \mu \mathrm{M}(\mathbf{b}-\mathbf{e})$

presented here suggest that ductal paucity in ALGS might also be due to compromised duct lineage specification and to decreased Jagged signaling from endoderm-derived cells in the liver and pancreas. Jag1 heterozygous mice exhibit a liver duct paucity defect only when they are extensively back-crossed into a C57BL/6 J background ${ }^{59}$, suggesting that other genes are involved. Consistently, Jag1 and Notch2 double heterozygous mice do exhibit biliary paucity ${ }^{60}$. However, this defect was attributed to a duct morphogenesis failure rather than a failure in cell fate specification ${ }^{32}$. Although the contrast between our finding of a duct lineage specification defect due to loss of both Jag1b and Jag2b Notch ligands and the reported duct morphogenesis defect with loss of Jag1 is likely a result of differential levels or timing of Jagged loss-of-function, speciesspecific differences cannot be ruled out. Future knockout of both Jag1 and Jag2 from mouse hepatopancreatic progenitors could reveal a conserved role for Jagged ligands in duct lineage specification in mammals. If compromised duct lineage specification is indeed a contributing factor to ductal paucity in ALGS patients, we will need to rethink the therapeutic strategies for this pathology and consider a regenerative approach to replace lost duct cells in this disease. 


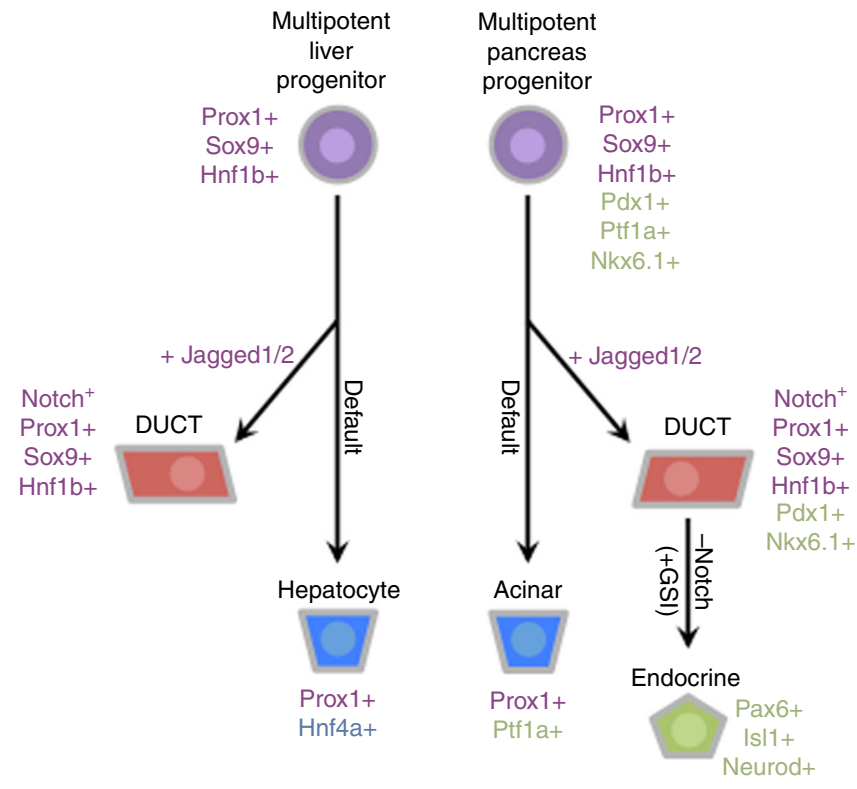

Fig. 6 Model diagram depicting common role for Jagged1b and Jagged2b in lineage specification of liver and pancreas ducts in zebrafish. During organogenesis, multipotent liver and pancreas progenitors, by default, give rise to hepatocytes and acinar cells, respectively. Jagged/Notch signaling is required in both organs for induction of duct lineage specification from these multipotent precursors. Once Notch signaling is activated in a cell, it contributes exclusively to the duct lineage. In the pancreas, loss of Notch signaling via gamma secretase inhibitors (GSI) from lineage specified duct cells could lead to their mis-differentiation into endocrine cells. Factors listed in purple demonstrate genetic similarities of cell types between the pancreas and liver. In green and blue are factors specific to the pancreas and liver, respectively

\section{Methods}

Zebrafish strains. Zebrafish were raised and maintained under standard conditions. The following mutants and transgenic lines were used: jag1b $b^{b 110538}$; jag2 $b^{\text {hu3 } 3425}, n$ pasl $4^{s 5}$ (cloche $)^{55}, \operatorname{Tg}\left(T p 1\right.$ loglob:eGFP) ${ }^{\text {um1439 }}$, abbreviated as Tg(Tp1:GFP); Tg(ptf1a:GFP) ${ }^{\text {jh161; }}$ Tg(lfabp10:DsRed, elaA:EGFP)gz1262, 63.

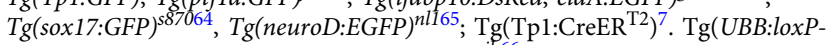
CFP-STOP-Terminator-loxP-hmgb1-mCherry jh66 transgenic fish were made from a transgene modified from the $\beta$-actin:loxP-CFP-loxP-hmgbl-mCherry construct. The $\beta$-actin promoter was replaced with promoter/enhancer sequence from ubiquitinB $(u b b)^{66}$. To minimize read through, three stop codons were added after the CFP open reading frame along with Cauliflower mosaic virus (CaMV) $35 \mathrm{~S}$ terminator sequence ${ }^{67}$ from plasmid pCSKalTA4GI ${ }^{68}$ and two SV40 polyA signals. This Cre-responder construct was injected with Tol2-transposon RNA to generate mosaic F0 fish ${ }^{69}$. Multiple F1 lines were screened for ubiquitous blue fluorescence and Cre-dependent nuclear red fluorescence. One line (jh63) was selected for further use as it displayed a high level of fluorescent marker expression along with high sensitivity to Cre recombination.

Immunohistochemistry. Mutant and wild-type siblings were anesthetized and washed for $1.5 \mathrm{~min}$ in PBS with .3\% Triton X-100 for permeabilization, then fixed with $2 \%$ formaldehyde in $0.1 \mathrm{M}$ PIPES, $1.0 \mathrm{mM} \mathrm{MgSO}_{4}$ and $2 \mathrm{mM}$ EGTA overnight at $4{ }^{\circ} \mathrm{C}$. The embryos were manually deyolked under a dissecting microscope and blocked for 1 hour in PBS with 4\% BSA and 0.3\% Triton X-100. Primary and secondary antibodies were incubated overnight. Washes were done after each step with $0.3 \%$ Triton X-100 in PBS for $3 \mathrm{~h}$ to remove excess antibodies. Antibodies used include mouse monoclonal anti-Nkx6.1 (1:20; Developmental Studies Hybridoma Bank (DSHB), Iowa), rabbit polyclonal anti-Prox1 (1:200; Millipore), goat polyclonal anti-Hnf4a (1:50, Santa Cruz), rabbit polyclonal antipan-Cadherin (1:5000; Sigma), mouse monoclonal anti-Islet1/2 (1:20; DSHB), mouse monoclonal 2F11 anti-Anxa4 ${ }^{41,70}$ (1:1000; gift from J. Lewis, Cancer Research UK), mouse monoclonal anti-Alcama (1:20; DSHB), mouse anti-ZO1 (1:200, Zymed), chick or rabbit polyclonal anti-GFP (1:300; Torrey Pines Biolabs), rabbit anti-cleaved Caspase-3 (1:50; Cell Signaling). Imaging was done with a Zeiss 710 confocal microscope with Zen9 software and images were processed with Adobe Photoshop CS3.
Lineage tracing and cell counting. For $\mathrm{CreER}^{\mathrm{T} 2}$ induction, embryos were incubated in 20uM 4-Hydroxytamoxifen (4-OHT, T176, Sigma, St. Louis, MO) for $2 \mathrm{~h}$ pulses, beginning at $42 \mathrm{hpf}$ every $12 \mathrm{~h}$ until $72 \mathrm{hpf} 4-\mathrm{OHT}$ was dissolved in $100 \%$ DMSO for a stock solution of $10 \mathrm{mM}$, and dissolved in embryo media. For quantification of lineage traced cells, embryos were stained for Hnf4a, Prox1, mCherry and DAPI for liver analysis; and Nkx6.1, mCherry and DAPI for pancreas, with either Elastase or ptfla:eGFP, for pancreatic analysis. mCherry positive cells were manually counted using Zen9 software in confocal z-stack images of whole livers and pancreas. To quantify endocrine cell number in jagged mutants, offspring embryos from jag $1 b^{-/+} ; j a g 2 b^{-/+}$parents were fixed at $87 \mathrm{hfp}$, stained for Pdx1, Nkx6.1 and DNA, and imaged using confocal microscopy. Total Islet ${ }^{+}$cells inside and outside the primary Islet were manually counted from z-stack images, and individual samples thereafter genotyped as described above. Control and experimental samples were scored blinded when possible.

Whole-mount fluorescent in situ hybridization. For whole-mount ISH, zebrafish embryos were fixed in 4\% paraformaldehyde (PFA) and dehydrated in methanol Rehydrated embryos were deyolked under a bright-field dissecting microscope, treated with $1.5 \mu \mathrm{g} / \mathrm{ml}$ proteinase $\mathrm{K}$ for 75 or $90 \mathrm{~min}$ for 56 and $72 \mathrm{hpf}$ embryos, respectively, post-fixed with $4 \%$ PFA for 20 min, then washed four times in phosphate-buffered saline with $0.1 \%$ Tween-20 (PBST). The prepared embryos were then pre-hybridized ( $50 \%$ formamide, $5 \mathrm{x}$ saline sodium citrate (SSC) buffer, $100 \mu \mathrm{g} / \mathrm{ml}$ yeast RNA, $50 \mu \mathrm{g} / \mathrm{ml}$ heparin, $0.125 \%$ Tween-20; $\mathrm{pH}$ to 6.0 with citric acid) at $68.5^{\circ} \mathrm{C}$ for four hours and then incubated overnight with jaglb and jag $2 b$ probes $^{38}$. The following day, embryos were subjected to stringency washes in a formamide/SSC series, washed twice in PBST at room temperature, treated with $2 \%$ $\mathrm{H}_{2} \mathrm{O}_{2}$ in PBST for one hour, washed four times in TNT buffer $(100 \mathrm{mM}$ Tris- $\mathrm{HCl}, \mathrm{pH} 7.5,150 \mathrm{mM} \mathrm{NaCl}, 0.1 \%$ Tween-20), and blocked in $0.5 \%$ Perkin Elmer blocking reagent in TNT for four hours. After incubating overnight with an anti-DIG-horseradish peroxidase antibody (1:400; Roche) at $4^{\circ} \mathrm{C}$, the embryos were washed eight times in TNT, pre-treated with PE Amplification Diluent for 5 minutes, and then exposed to TSA Cyanine3 (1:60; Perkin Elmer) for one hour. The reaction was quenched by treatment with $2 \% \mathrm{H}_{2} \mathrm{O}_{2}$ in TNT for one hour, and the embryos were washed in PBS and immunostained with a rabbit anti-GFP antibody (1:1000; Torrey Pines Biolabs) and an Alexa488conjugated goat anti-rabbit secondary antibody (1:300; Molecular Probes). The DRAQ5 nuclear stain (1:500; BioStatus) was applied with the secondary antibody.

Morpholino injection and endoderm transplantation. Translation blocking morpholinos for jag1b (5'-CTGAACTCCGTCGCAGAATCATGCC-3') and jag $2 b$ (5'- TCCTGATACAATTCCACATGCCGCC- $3^{\prime 34}$ (Gene Tools) were combined and diluted in $0.2 \mathrm{M} \mathrm{KCl}$ and phenol red from a $8.5 \mathrm{mg} / \mathrm{ml}$ stock to $2 \mathrm{mg} / \mathrm{ml}$ (each) working concentration. One nanoliter was injected for a final amount of 2 nanograms each into the host embryos. Donors embryos were injected with 100 picograms of sox32 mRNA, and rhodamine dextran at the one cell stage. At $4 \mathrm{hpf}$, both donor and host embryos were dechorionated in an agarose-coated petri dish. Donor cells were drawn into a glass needle (pulled from Borosilicate Glass Capillaries, World Precision Instruments, Inc.) using CellTram Air (Eppendorf). About 15 donor cells were then injected into one host embryo using CellTram Air.

Chemical inhibitor treatment. Notch signaling was inhibited at various stages of pancreas development using a gamma-secretase inhibitor (GSI), LY411575 ${ }^{50}$. Larvae were treated with $50 \mu \mathrm{M}$ LY411575 (Sigma) in egg water with $1 \% \mathrm{DMSO}^{50}$ Sibling larvae from the same clutch were treated with $1 \%$ DMSO in egg water as control. Embryos were treated from 28-132 hpf for early inhibition, and from $69 \mathrm{hpf}-132 \mathrm{hpf}$ for late inhibition.

Statistical analysis. Calculations were made in Microsoft Excel. We report mean and standard deviation, the probability associated with the Student's t test (with two-tailed distribution). $P$ values were calculated by Student's $t$-test for single comparisons of normally distributed data. $P$-value was obtained through comparison of data sets: the expected number of acinar or hepatocyte cells labeled verses the actual number of acinar or hepatocyte cells labeled. Expected number of acinar and hepatocytes were based on Cre efficiency multiplied by a conservative estimates of acinar:ductal cell ratio of $3: 1^{71}$ and hepatocyte:ductal cell ratio of $6: 1^{40}$ We used GraphPad online software to perform Student's t test (with two-tail distribution). The two-tailed $P$ values are less than 0.0001 . By conventional criteria, this difference is considered to be extremely statistically significant.

Data availability. The authors declare that all data supporting the findings of this study are available within the article and its Supplementary Information Files or from the corresponding author upon reasonable request.

Received: 19 December 2016 Accepted: 15 July 2017

Published online: 03 October 2017 


\section{References}

1. Danks, D. M., Campbell, P. E., Jack, I., Rogers, J. \& Smith, A. L. Studies of the aetiology of neonatal hepatitis and biliary atresia. Arch. Dis. Child. 52, 360-367 (1977).

2. Li, L. et al. Alagille syndrome is caused by mutations in human Jagged1, which encodes a ligand for Notch1. Nat. Genet. 16, 243-251 (1997).

3. McDaniell, R. et al. NOTCH2 mutations cause Alagille syndrome, a heterogeneous disorder of the notch signaling pathway. Am. J. Hum. Genet. 79, 169-173 (2006)

4. Oda, T. et al. Mutations in the human Jagged1 gene are responsible for Alagille syndrome. Nat. Genet. 16, 235-242 (1997).

5. Turnpenny, P. D. \& Ellard, S. Alagille syndrome: pathogenesis, diagnosis and management. Eur. J. Hum. Genet. 20, 251-257 (2012).

6. Furuyama, K. et al. Continuous cell supply from a Sox9-expressing progenitor zone in adult liver, exocrine pancreas and intestine. Nat. Genet. 43, 34-41 (2011).

7. Choi, T. Y., Ninov, N., Stainier, D. Y. \& Shin, D. Extensive conversion of hepatic biliary epithelial cells to hepatocytes after near total loss of hepatocytes in zebrafish. Gastroenterology. 146, 776-788 (2014).

8. Font-Burgada, J. et al. Hybrid periportal hepatocytes regenerate the injured liver without giving rise to cancer. Cell 162, 766-779 (2015).

9. Lu, W. Y. et al. Hepatic progenitor cells of biliary origin with liver repopulation capacity. Nat. Cell. Biol. 17, 971-983 (2015).

10. Schaub, J. R., Malato, Y., Gormond, C. \& Willenbring, H. Evidence against a stem cell origin of new hepatocytes in a common mouse model of chronic liver injury. Cell Rep 8, 933-939 (2014).

11. Tarlow, B. D., Finegold, M. J. \& Grompe, M. Clonal tracing of Sox9+liver progenitors in mouse oval cell injury. Hepatology. 60, 278-289 (2014).

12. Yanger, K. et al. Adult hepatocytes are generated by self-duplication rather than stem cell differentiation. Cell. Stem. Cell. 15, 340-349 (2014).

13. Carpentier, R. et al. Embryonic ductal plate cells give rise to cholangiocytes, periportal hepatocytes, and adult liver progenitor cells. Gastroenterology. 141, 1432-1438 (2011)

14. Kopp, J. L. et al. Sox9+ductal cells are multipotent progenitors throughout development but do not produce new endocrine cells in the normal or injured adult pancreas. Development 138, 653-665 (2011)

15. Solar, M. et al. Pancreatic exocrine duct cells give rise to insulin-producing beta cells during embryogenesis but not after birth. Dev. Cell. 17, 849-860 (2009).

16. Antoniou, A. et al. Intrahepatic bile ducts develop according to a new mode of tubulogenesis regulated by the transcription factor SOX9. Gastroenterology. 136, 2325-2333 (2009).

17. Seymour, P. A. et al. SOX9 is required for maintenance of the pancreatic progenitor cell pool. Proc. Natl Acad. Sci. USA 104, 1865-1870 (2007).

18. Dong, P. D., Provost, E., Leach, S. D. \& Stainier, D. Y. Graded levels of Ptfla differentially regulate endocrine and exocrine fates in the developing pancreas. Genes Dev. 22, 1445-1450 (2008).

19. Gradwohl, G., Dierich, A., LeMeur, M. \& Guillemot, F. neurogenin3 is required for the development of the four endocrine cell lineages of the pancreas. Proc. Natl Acad. Sci. USA 97, 1607-1611 (2000).

20. Afelik, S. \& Jensen, J. Notch signaling in the pancreas: patterning and cell fate specification. Wiley Interdiscip Rev Dev. Biol. 2, 531-544 (2013).

21. Geisler, F. \& Strazzabosco, M. Emerging roles of Notch signaling in liver disease. Hepatology. 61, 382-392 (2015).

22. Hald, J. et al. Activated Notch1 prevents differentiation of pancreatic acinar cells and attenuate endocrine development. Dev. Biol. 260, 426-437 (2003).

23. Tchorz, J. S. et al. Notch2 signaling promotes biliary epithelial cell fate specification and tubulogenesis during bile duct development in mice. Hepatology. 50, 871-879 (2009).

24. Horn, S. et al. Mind bomb 1 is required for pancreatic beta-cell formation. Proc. Natl Acad. Sci. USA 109, 7356-7361 (2012).

25. Itoh, M. et al. Mind bomb is a ubiquitin ligase that is essential for efficient activation of Notch signaling by Delta. Dev. Cell. 4, 67-82 (2003).

26. Turkoz, M., Townsend, R. R. \& Kopan, R. The notch intracellular domain has an $\mathrm{RBPj}$-independent role during mouse hair follicular development. J. Invest. Dermatol. 136, 1106-1115 (2016).

27. Johnson, J. E. \& Macdonald, R. J. Notch-independent functions of CSL. Curr. Top. Dev. Biol. 97, 55-74 (2011).

28. Berndt, J. D. et al. Mindbomb 1, an E3 ubiquitin ligase, forms a complex with RYK to activate Wnt/beta-catenin signaling. J. Cell. Biol. 194, 737-750 (2011).

29. Kobayashi, T. \& Kageyama, R. Expression dynamics and functions of Hes factors in development and diseases. Curr. Top. Dev. Biol. 110, 263-283 (2014).

30. Saint Just Ribeiro, M. \& Wallberg, A. E. Transcriptional mechanisms by the coregulator MAML1. Curr. Protein. Pept. Sci. 10, 570-576 (2009).

31. Andersen, P., Uosaki, H., Shenje, L. T. \& Kwon, C. Non-canonical Notch signaling: emerging role and mechanism. Trends Cell Biol. 22, 257-265 (2012).

32. Lozier, J., McCright, B. \& Gridley, T. Notch signaling regulates bile duct morphogenesis in mice. PLoS. ONE. 3, e1851 (2008).
33. Hofmann, J. J. et al. Jagged1 in the portal vein mesenchyme regulates intrahepatic bile duct development: insights into Alagille syndrome. Development 137, 4061-4072 (2010).

34. Lorent, K. et al. Inhibition of Jagged-mediated Notch signaling disrupts zebrafish biliary development and generates multi-organ defects compatible with an Alagille syndrome phenocopy. Development 131, 5753-5766 (2004).

35. Zecchin, E. et al. Distinct delta and jagged genes control sequential segregation of pancreatic cell types from precursor pools in zebrafish. Dev. Biol. 301, 192-204 (2007)

36. Kok, F. O. et al. Reverse genetic screening reveals poor correlation between morpholino-induced and mutant phenotypes in zebrafish. Dev. Cell. 32, 97-108 (2015).

37. Howe, K. et al. The zebrafish reference genome sequence and its relationship to the human genome. Nature. 496, 498-503 (2013).

38. Zuniga, E., Stellabotte, F. \& Crump, J. G. Jagged-Notch signaling ensures dorsal skeletal identity in the vertebrate face. Development 137, 1843-1852 (2010).

39. Parsons, M. J. et al. Notch-responsive cells initiate the secondary transition in larval zebrafish pancreas. Mech. Dev. 126, 898-912 (2009).

40. Lorent, K., Moore, J. C., Siekmann, A. F., Lawson, N. \& Pack, M. Reiterative use of the notch signal during zebrafish intrahepatic biliary development. Dev. Dyn. 239, 855-864 (2010).

41. Zhang, D. et al. Identification of Annexin A4 as a hepatopancreas factor involved in liver cell survival. Dev. Biol. 395, 96-110 (2014).

42. Nelson, S. B., Schaffer, A. E. \& Sander, M. The transcription factors Nkx6.1 and Nkx6.2 possess equivalent activities in promoting beta-cell fate specification in Pdx1+pancreatic progenitor cells. Development 134, 2491-2500 (2007).

43. Seth, A. et al. Proxl ablation in hepatic progenitors causes defective hepatocyte specification and increases biliary cell commitment. Development 141, 538-547 (2014).

44. Wang, Y. et al. Moesin1 and Ve-cadherin are required in endothelial cells during in vivo tubulogenesis. Development 137, 3119-3128 (2010).

45. Ghaye, A. P. et al. Progenitor potential of nkx6.1-expressing cells throughout zebrafish life and during beta cell regeneration. BMC. Biol. 13, 70 (2015).

46. Schaffer, A. E., Freude, K. K., Nelson, S. B. \& Sander, M. Nkx6 transcription factors and Ptfla function as antagonistic lineage determinants in multipotent pancreatic progenitors. Dev. Cell. 18, 1022-1029 (2010)

47. Dong, P. D. et al. Fgf10 regulates hepatopancreatic ductal system patterning and differentiation. Nat. Genet. 39, 397-402 (2007).

48. Golson, M. L. et al. Jagged1 is a competitive inhibitor of Notch signaling in the embryonic pancreas. Mech. Dev. 126, 687-699 (2009).

49. Shih, H. P. et al. A Notch-dependent molecular circuitry initiates pancreatic endocrine and ductal cell differentiation. Development 139, 2488-2499 (2012).

50. Ninov, N., Borius, M. \& Stainier, D. Y. Different levels of Notch signaling regulate quiescence, renewal and differentiation in pancreatic endocrine progenitors. Development 139, 1557-1567 (2012)

51. Jurisch-Yaksi, N., Sannerud, R. \& Annaert, W. A fast growing spectrum of biological functions of gamma-secretase in development and disease. Biochim. Biophys. Acta. 1828, 2815-2827 (2013).

52. Ninov, N. et al. Metabolic regulation of cellular plasticity in the pancreas. Curr Biol. 23, 1242-1250 (2013).

53. Wang, Y., Rovira, M., Yusuff, S. \& Parsons, M. J. Genetic inducible fate mapping in larval zebrafish reveals origins of adult insulin-producing beta-cells Development 138, 609-617 (2011).

54. Reischauer, $\mathrm{S}$. et al. Cloche is a bHLH-PAS transcription factor that drives haemato-vascular specification. Nature. 535, 294-298 (2016).

55. Sakaguchi, T. F., Sadler, K. C., Crosnier, C. \& Stainier, D. Y. Endothelial signals modulate hepatocyte apicobasal polarization in zebrafish. Curr. Biol. 18, 1565-1571 (2008).

56. Zong, Y. et al. Notch signaling controls liver development by regulating biliary differentiation. Development 136, 1727-1739 (2009).

57. Stafford, D. et al. Retinoids signal directly to zebrafish endoderm to specify insulin-expressing beta-cells. Development 133, 949-956 (2006).

58. Shih, H. P., Wang, A. \& Sander, M. Pancreas organogenesis: from lineage determination to morphogenesis. Annu. Rev. Cell Dev. Biol. 29, 81-105 (2013)

59. Thakurdas, S. M. et al. Jagged1 heterozygosity in mice results in a congenital cholangiopathy which is reversed by concomitant deletion of one copy of Poglutl (Rumi). Hepatology. 63, 550-565 (2016).

60. McCright, B., Lozier, J. \& Gridley, T. A mouse model of Alagille syndrome: Notch2 as a genetic modifier of Jag1 haploinsufficiency. Development 129 1075-1082 (2002).

61. Godinho, L. et al. Targeting of amacrine cell neurites to appropriate synaptic laminae in the developing zebrafish retina. Development 132, 5069-5079 (2005).

62. Pisharath, H., Rhee, J. M., Swanson, M. A., Leach, S. D. \& Parsons, M. J. Targeted ablation of beta cells in the embryonic zebrafish pancreas using E. coli nitroreductase. Mech. Dev. 124, 218-229 (2007). 
63. Korzh, S. et al. Requirement of vasculogenesis and blood circulation in late stages of liver growth in zebrafish. BMC. Dev. Biol. 8, 84 (2008).

64. Mizoguchi, T., Verkade, H., Heath, J. K., Kuroiwa, A. \& Kikuchi, Y. Sdf1/ Cxcr4 signaling controls the dorsal migration of endodermal cells during zebrafish gastrulation. Development 135, 2521-2529 (2008).

65. Obholzer, N. et al. Vesicular glutamate transporter 3 is required for synaptic transmission in zebrafish hair cells. J. Neurosci. 28, 2110-2118 (2008).

66. Mosimann, C. et al. Ubiquitous transgene expression and Cre-based recombination driven by the ubiquitin promoter in zebrafish. Development 138, 169-177 (2011).

67. Hirt, H., Kogl, M., Murbacher, T. \& Heberle-Bors, E. Evolutionary conservation of transcriptional machinery between yeast and plants as shown by the efficient expression from the CaMV $35 \mathrm{~S}$ promoter and $35 \mathrm{~S}$ terminator. Curr. Genet. 17, 473-479 (1990).

68. Distel, M., Wullimann, M. F. \& Koster, R. W. Optimized Gal4 genetics for permanent gene expression mapping in zebrafish. Proc. Natl Acad. Sci. USA 106, 13365-13370 (2009).

69. Kawakami, K. et al. A transposon-mediated gene trap approach identifies developmentally regulated genes in zebrafish. Dev. Cell. 7, 133-144 (2004).

70. Crosnier, C. et al. Delta-Notch signalling controls commitment to a secretory fate in the zebrafish intestine. Development 132, 1093-1104 (2005).

71. Wang, Y. J., Park, J. T., Parsons, M. J. \& Leach, S. D. Fate mapping of ptfla-expressing cells during pancreatic organogenesis and regeneration in zebrafish. Dev. Dyn. 244, 724-735 (2015).

\section{Acknowledgements}

We thank the zebrafish research community for the numerous reagents shared, including Nikolay Ninov for the Tp1:Cre reporter line, and members of our laboratory for critical discussions. We thank and apologize to authors whose work we were not able to cite due to space constraints. We thank Alexey Eroshkin of the Sanford Burnham Prebys Bioinformatics Core for help with statistical and quantitative analysis. This work was supported by funds from National Institutes of Health (NIH) 1DP2DK098092 to P.D.S.D., NIH R01 DE018405 to J.G.C., NIH P30 DK048522 to Crump, J.G.C., NIH 4R01DK080730-09 to M.J.P., CIRM TG2-01168 to M.G.

\section{Author contributions}

D.Z. and K.P.G designed and executed experiments, and assembled figures with the guidance of P.D.S.D.; P.D.S.D. oversaw studies; L.B. performed whole mount fluorescent in situ hybridization; L.B., M.G., K.W. and J.G.C. independently characterized Notch reporter activity in jagged mutant embryos; G.W. and M.J.P. generated transgenic Cre reporter zebrafish line; X-X.I.Z performed statistical analysis. P.D.S.D., D.Z., and K.P.G. wrote manuscript with critical feedback from J.G.C., M.J.P., J.J.L., and L.B.

\section{Additional information}

Supplementary Information accompanies this paper at doi:10.1038/s41467-017-00666-6.

Competing interests: The authors declare no competing financial interests.

Reprints and permission information is available online at http://npg.nature.com/ reprintsandpermissions/

Publisher's note: Springer Nature remains neutral with regard to jurisdictional claims in published maps and institutional affiliations.

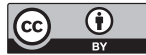

Open Access This article is licensed under a Creative Commons Attribution 4.0 International License, which permits use, sharing, adaptation, distribution and reproduction in any medium or format, as long as you give appropriate credit to the original author(s) and the source, provide a link to the Creative Commons license, and indicate if changes were made. The images or other third party material in this article are included in the article's Creative Commons license, unless indicated otherwise in a credit line to the material. If material is not included in the article's Creative Commons license and your intended use is not permitted by statutory regulation or exceeds the permitted use, you will need to obtain permission directly from the copyright holder. To view a copy of this license, visit http://creativecommons.org/ licenses/by/4.0/.

(C) The Author(s) 2017 